\title{
Treatment of Natural Geometry in Finite Volume River Flow Computations
}

\author{
H. Capart ${ }^{1}$; T. I. Eldho'; S. Y. Huang ${ }^{3}$; D. L. Young ${ }^{4}$; and Y. Zech ${ }^{5}$
}

\begin{abstract}
A method is proposed for the treatment of irregular bathymetry in one-dimensional finite volume computations of openchannel flow. The strategy adopted is based on a reformulation of the Saint-Venant equations. In contrast with the usual treatment of topography effects as source terms, the method accounts for slope and nonprismaticity by modifying the momentum flux. This makes it possible to precisely balance the hydrostatic pressure contributions associated with variations in valley geometry. The characteristic method is applied to the revised equations, yielding topographic corrections to the numerical fluxes of an upwind scheme. Further adaptations endow the scheme with an ability to capture transcritical sections and wetting fronts in channels of abrupt topography. To test the approach, the scheme is first applied to idealized benchmark problems. The method is then used to route a severe flood through a complex river system: the Tanshui in Northern Taiwan. Computational results compare favorably with gauge records. Discrepancies in water stage represent no more than a fraction of the magnitude of typical bathymetry variations.
\end{abstract}

DOI: $10.1061 /(A S C E) 0733-9429(2003) 129: 5(385)$

CE Database subject headings: Open-channel flow; Algorithms; Geometry; Flood routing; River systems.

\section{Introduction}

First introduced in hydraulics more than a decade ago (Fennema and Chaudhry 1987; Glaister 1988; for a review, see Chaudhry 1993), finite volume schemes have evolved in recent years into powerful tools for the prediction of shallow water flows (Alcrudo et al. 1992; Braschi and Gallati 1992; Toro 1992; Glaister 1993; Yang et al. 1993; Jha et al. 1996; Jin and Fread 1997; Sanders and Katopodes 1999; Tseng and Chu 2000; Sanders 2001). Among other attractive features, these algorithms are noteworthy for their ability to deal with transcritical flows and propagating shocks. Favorable comparisons with analytical benchmarks and laboratory measurements have been obtained for complex flows in oneand two-dimensional configurations (e.g., Fraccarollo and Toro 1995; Capart et al. 1997; Soares et al. 2000; Tseng et al. 2001; Bradford and Sanders 2002 ). Despite these good performances, finite volume algorithms have not yet found wide acceptance in the context of practical river studies. In no small measure, this is due to difficulties associated with the bathymetry of natural val-

\footnotetext{
${ }^{1}$ Research Fellow, Fonds National de la Recherche Scientifique and Univ. catholique de Louvain, Belgium.

${ }^{2}$ Assistant Professor, Dept. of Civil Engineering, Indian Institute of Technology, Bombay, Mumbai, India - 400076.

${ }^{3}$ Graduate Student, Dept. of Civil Engineering and Hydrotech Res. Institute, National Taiwan Univ., 258 Chow-Shan Rd., Taipei 10617, Taiwan.

${ }^{4}$ Professor, Dept. of Civil Engineering and Hydrotech Res. Institute, National Taiwan Univ., 258 Chow-Shan Rd., Taipei 10617, Taiwan (corresponding author). E-mail: dlyoung@hy.ntu.edu.tw.

${ }^{5}$ Professor, Dept. of Civil Engineering, Univ. catholique de Louvain, Belgium.

Note. Discussion open until October 1, 2003. Separate discussions must be submitted for individual papers. To extend the closing date by one month, a written request must be filed with the ASCE Managing Editor. The manuscript for this paper was submitted for review and possible publication on August 10, 2000; approved on November 19, 2002. This paper is part of the Journal of Hydraulic Engineering, Vol. 129, No. 5, May 1, 2003. ()ASCE, ISSN 0733-9429/2003/5-385-393/\$18.00.
}

leys. The core problem is well-understood (Bermudez and Vazquez 1994; Ambrosi 1995; Nujic 1995): Over irregular topography, momentum- conserving algorithms tend to balance incorrectly the hydrostatic pressure contributions acting on the lateral and bottom boundaries of the finite volumes. Solutions to this problem have been proposed for prismatic channels of variable bed profile (Bermudez and Vasquez 1994; Nujic 1995), and for nonprismatic channels of regular geometry (Meselhe et al. 1997; Tseng et al. 2001; Sanders 2001). The present work addresses the more general case of inclined, nonprismatic channels characterized by abrupt variations in bathymetry. A simple method is sought, suitable for practical applications in which information about the valley geometry is given as irregular transect data.

In the context of one-dimensional (1D) finite volume algorithms, the Saint-Venant equations describing unsteady openchannel flows are generally written in the form (Cunge et al. 1980)

$$
\begin{gathered}
\frac{\partial A}{\partial t}+\frac{\partial Q}{\partial x}=0 \\
\frac{\partial Q}{\partial t}+\frac{\partial}{\partial x}\left(\frac{Q^{2}}{A}+g I_{1}\right)=g A\left(S_{0}-S_{f}\right)+g I_{2}
\end{gathered}
$$

where $t=$ time; $x=$ streamwise coordinate; $A=$ wetted crosssectional area; $Q=$ discharge; $g=$ gravitational acceleration; and $S_{f}=$ friction slope typically modeled by Manning's equation. Terms $S_{0}, I_{1}$, and $I_{2}$ depend on the channel geometry and are given by

$$
\begin{gathered}
S_{0}=-\frac{\partial z_{0}}{\partial x} \\
I_{1}=\int_{0}^{h}(h-\eta) b(x, \eta) d \eta \\
I_{2}=\int_{0}^{h}(h-\eta) \frac{\partial b(x, \eta)}{\partial x} d \eta
\end{gathered}
$$




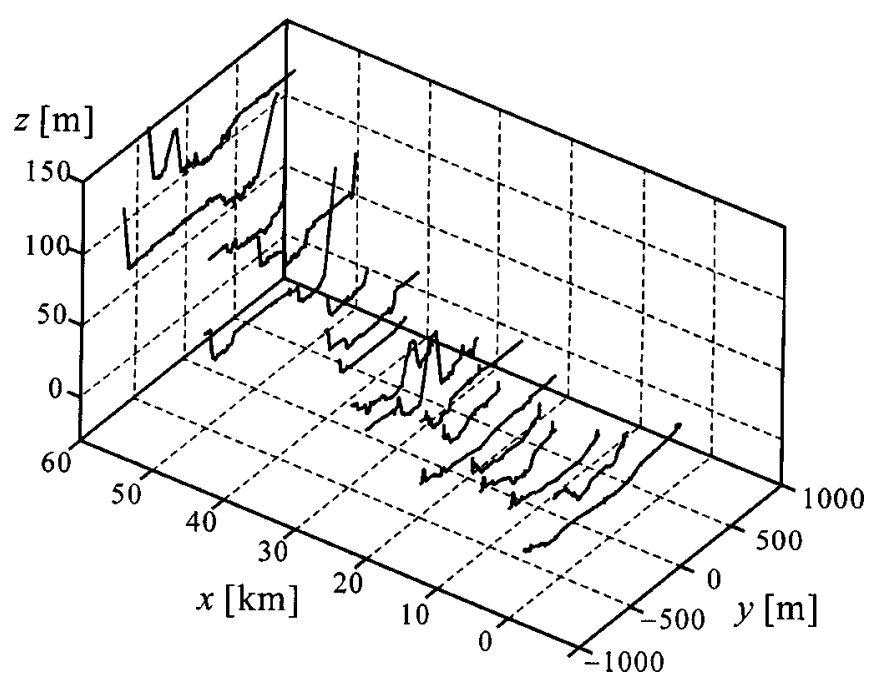

Fig. 1. Channel geometry represented by irregular cross-section data. Selected transects (1 in 5) for Tahan Creek and Tanshui River, Northern Taiwan: $x=$ distance from river mouth; $y=$ transverse coordinate with respect to thalweg; $z=$ elevation above mean sea level.

where $S_{0}=$ bottom slope; $I_{1}=$ first moment of the wetted cross section with respect to the free surface; $I_{2}=$ spatial variation of the first moment; and $h=$ flow depth. In the above expressions, the channel geometry is represented by bottom elevation profile $z_{0}(x)$ and width-depth relationship $b(x, \eta)$. Continuity Eq. (1) represents conservation of water volume, while Eq. (2) expresses balance of horizontal momentum. Under the shallow water assumption, hydrostatic pressure is assumed both within the water body and along its solid boundaries.

For frictionless, prismatic, horizontal channels, the source terms on the right-hand side of Eq. (2) drop out and the remaining equations are in full conservation form. They admit weak solutions composed of piecewise smooth profiles connected by shocklike discontinuities representing hydraulic jumps and bores. The effects of channel inclination and nonprismatic geometry are included in $S_{0}$ and $I_{2}$, which involve spatial gradients $\partial z_{0} / \partial x$ and $\partial b / \partial x$. For these terms to be well defined, the channel geometry must everywhere be gradually varied. It is this condition which proves too restrictive in many practical situations. In actual river computations, the channel geometry is typically described by a series of transects oriented in a roughly perpendicular direction with respect to the main thalweg. As illustrated in Fig. 1 for the Tanshui River in Taiwan, the resulting cross-sectional data can be highly irregular. This reflects both the intrinsic variability of the valley topography and the approximate character of surveying procedures. In such circumstances, spatial gradients $\partial z_{0} / \partial x$ and $\partial b / \partial x$ are nowhere easily defined and direct application of Eq. (2) is not feasible. One possible approach is to regularize the crosssection data and obtain simpler, more regular transects (e.g., trapezoidal profiles) to which the formulation applies. The alternative developed in the present work is to seek a more robust version of Eq. (2) suitable for the direct application of finite volume schemes to irregular valley data. Such a revised momentum equation is presented in the first section of the present paper. It is exploited in a second section to derive topographic corrections to the upwind finite volume scheme of Braschi and Gallati (1992). Validation tests are covered in a third section. Finally, the proposed strategy is applied to a field case: The routing of a typhoon flood through the Tanshui River network in Northern Taiwan.

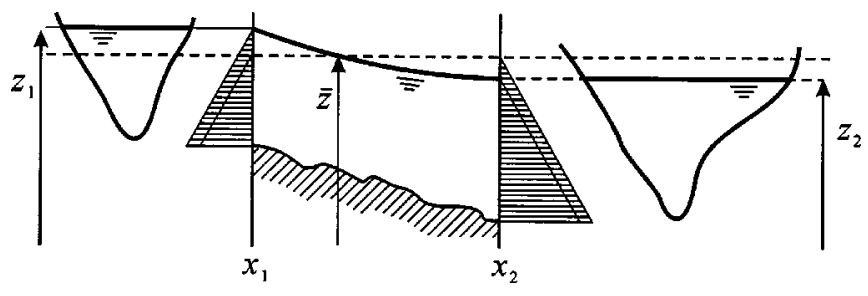

Fig. 2. Hydrostatic balance of channel reach featuring irregular bathymetry

\section{Approximate Momentum Balance for Irregular Geometry}

With reference to Fig. 2, consider a channel reach comprised between transverse cross sections of known geometry surveyed at longitudinal positions $x_{1}$ and $x_{2}$. Application of momentum balance to this control volume yields the following integral equation:

$$
\frac{\partial}{\partial t} \int_{x_{1}}^{x_{2}} Q d x+\left[\frac{Q^{2}}{A}\right]_{x_{1}}^{x_{2}}+\frac{1}{\rho} \int_{\Gamma} p n_{x} d \Gamma=-\int_{x_{1}}^{x_{2}} g A S_{f} d x
$$

where $[f]_{x_{1}}^{x_{2}}=f\left(x_{2}\right)-f\left(x_{1}\right) ; \quad \rho=$ mass density of water; $p=$ pressure $\Gamma=$ control surface; and $n_{x}=x$-component of the outward normal to $\Gamma$. As the bottom boundary $\Gamma_{0}$ is rigid, the flux of convected inertia is nonzero only through the left and right vertical faces $\Gamma_{1}, \Gamma_{2}$ of control volume $\Omega$, and reduces to $\left[Q^{2} / A\right]_{x_{1}}^{x_{2}}$. The third term on the left-hand side of Eq. (4) represents the overall pressure thrust acting on control surface $\Gamma$. This integral can be decomposed into three separate contributions

$$
\begin{aligned}
\int_{\Gamma} p n_{x} d \Gamma & =\int_{\Gamma_{1}} p n_{x} d \Gamma+\int_{\Gamma_{2}} p n_{x} d \Gamma+\int_{\Gamma_{0}} p n_{x} d \Gamma \\
& =\left[\rho g I_{1}\right]_{x_{1}}^{x_{2}}+\int_{\Gamma_{0}} p n_{x} d \Gamma
\end{aligned}
$$

corresponding to integrals of the hydrostatic pressure over the left and right vertical faces $\Gamma_{1}, \Gamma_{2}$, to which is added the hydrostatic pressure thrust exerted along the bottom boundary $\Gamma_{0}$. Rigorous evaluation of this last contribution requires knowledge of the bottom topography $\Gamma_{0}$ between $x_{1}$ and $x_{2}$. Our objective is to seek an approximation for this term, relying solely on prescribed crosssectional profiles at locations $x_{1}$ and $x_{2}$. Let $\bar{z}=1 / 2\left(z_{1}+z_{2}\right)$ denote an average elevation of the water surface within the control volume. In the case of a horizontal free surface, $z_{1}=z_{2}=\bar{z}$. From classical hydrostatics, the integral of hydrostatic pressure forces exerted on the boundary of a body of water presenting a horizontal free surface is equal to zero. No matter how complex the bathymetry, it follows that

$$
\left.\int_{\Gamma} p\right|_{\bar{z}} n_{x} d \Gamma=0
$$

where $\Gamma=\Gamma_{1} \cup \Gamma_{2} \cup \Gamma_{0}$; and $\left.p\right|_{\bar{z}}$ denotes the hydrostatic pressure obtained under a water surface elevation constrained equal to $\bar{z}$ throughout the control volume. Like Eq. (5), integral Eq. (6) can be decomposed into separate contributions

$$
\left.\int_{\Gamma} p\right|_{\bar{z}} n_{x} d \Gamma=\left[\left.\rho g I_{1}\right|_{\bar{z}}\right]_{x_{1}}^{x_{2}}+\left.\int_{\Gamma_{0}} p\right|_{\bar{z}} n_{x} d \Gamma
$$

where 


$$
\left.I_{1}\right|_{\bar{z}}=\int_{0}^{\bar{z}-z_{0}}\left(\bar{z}-z_{0}-\eta\right) b(x, \eta) d \eta
$$

is the first moment of the wetted cross section with respect to the constrained free surface elevation $\bar{z}$ (rather than the local free surface elevation $z$ ). Hydrostatic equilibrium constraint (6) can then be subtracted from pressure thrust integral (5) to yield

$$
\int_{\Gamma} p n_{x} d \Gamma=\left[\rho g I_{1}-\left.\rho g I_{1}\right|_{\bar{z}}\right]_{x_{1}}^{x_{2}}+\int_{\Gamma_{0}}\left(p-\left.p\right|_{\bar{z}}\right) n_{x} d \Gamma
$$

Assuming that the slope of the water surface (reflected by the pressure difference $p-\left.p\right|_{\bar{z}}$ ) is mild and that the streamwise component of the sidewall inclination (denoted by $n_{x}$ ) is small, the last term on the right-hand side of Eq. (9) is a product of two small quantities and can be neglected in first approximation. One is thus left with the approximate equivalence

$$
\int_{\Gamma} p n_{x} d \Gamma \approx\left[\rho g I_{1}-\rho g I_{1} \mid \bar{z}\right]_{x_{1}}^{x_{2}}
$$

This approximation is exact in two cases: When the channel is rectangular in shape and has a constant width (regardless of the slope of the water surface), and when the water surface is horizontal (regardless of the channel geometry). In the latter case, the hydrostatic pressure terms balance exactly: Hydrostatic equilibrium holds no matter whether the channel is inclined, nonprismatic, or arbitrarily irregular in shape. The main advantage of approximation (10) is that it does preserve this important equilibrium. Furthermore, estimate (10) involves the bottom topography only at the discrete cross-sections $x_{1}$ and $x_{2}$ where the survey data are available. Under approximation (10), integral Eq. (4) becomes

$$
\frac{\partial}{\partial t} \int_{x_{1}}^{x_{2}} Q d x+\left[\frac{Q^{2}}{A}+g I_{1}-\left.g I_{1}\right|_{\bar{z}}\right]_{x_{1}}^{x_{2}}=-\int_{x_{1}}^{x_{2}} g A S_{f} d x
$$

If discontinuities are absent within control volume $\Omega$, one can let $x_{2}-x_{1}=\Delta x \rightarrow 0$ and obtain Eq. (11) in the following divergence form:

$$
\frac{\partial Q}{\partial t}+\frac{\partial}{\partial x}\left(\frac{Q^{2}}{A}+g I_{1}-\left.g I_{1}\right|_{\bar{z}}\right)=-g A S_{f}
$$

which constitutes an alternative version of momentum Eq. (2). Formulation $\partial\left(g I_{1} \mid \bar{z}\right) / \partial x$ means that the differentiation is to be performed on the channel geometry, holding the reference free surface elevation constant and equal to level $\bar{z}$. What has been achieved is a transfer of channel inclination and nonprismatic geometry effects from the right-hand side of Eq. (2) to the lefthand side of Eq. (12). The effect of variable bathymetry is included in Eq. (2) as a source term, while in Eq. (12) it is incorporated within the divergence operator. This strategy is the opposite of the one adopted by Nujic (1995) to deal with an irregular bottom profile. In the work of this writer, hydrostatic balance is achieved by extracting pressure term $g \partial I_{1} / \partial x$ from the divergence operator and transferring it to the right-hand side of Eq. (2). Whereas the treatment of Nujic (1995) is restricted to prismatic channels of rectangular cross section, the present approach applies to the nonprismatic case as well. Eqs. (1) and (12) can now be taken as a basis for the formulation of a finite volume algorithm.

\section{Finite Volume Numerical Scheme}

\section{Basic Formulation}

We consider the following computational domain. Cross-sectional bathymetry data are provided at discrete locations $x_{i}$. In general, the $x_{i}$ are not equidistant. Vertical interfaces placed at positions $x_{i+1 / 2}=1 / 2\left(x_{i}+x_{i+1}\right)$ define distinct cells, inside which constant states $A_{i}, Q_{i}$ are assumed. A first-order accurate discretization of Eqs. (1) and (12) is then given by

$$
\begin{gathered}
A_{i}^{(m+1)}=A_{i}^{(m)}+\frac{\Delta t}{(\Delta x)_{i}}\left(Q_{i-1 / 2}^{*}-Q_{i+1 / 2}^{*}\right) \\
Q_{i}^{(m+1)}=Q_{i}^{(m)}+\frac{\Delta t}{(\Delta x)_{i}}\left(\Sigma_{i-1 / 2}^{*, \text { right }}-\Sigma_{i+1 / 2}^{*, \text { left }}\right)-\left(g A S_{f}\right)_{i}^{(m)} \Delta t
\end{gathered}
$$

where superscripts $(m)$ and $(m+1)=$ variables sampled at successive times $t^{(m)}, t^{(m+1)}$; superscript $*=$ intermediate state between $t^{(m)}$ and $t^{(m+1)} ; \Delta t=t^{(m+1)}-t^{(m)}$ and $(\Delta x)_{i}=x_{i+1 / 2}-x_{i-1 / 2}=$ the time and space intervals, and where we have introduced

$$
\begin{aligned}
& \sum_{i-1 / 2}^{*, \text { right }}=\left(\frac{Q^{2}}{A}+g I_{1}-\left.g I_{1}\right|_{z_{i}}\right)_{i-1 / 2}^{*} \\
& \Sigma_{i+1 / 2}^{*, \text { left }}=\left(\frac{Q^{2}}{A}+g I_{1}-\left.g I_{1}\right|_{z_{i}}\right)_{i+1 / 2}^{*}
\end{aligned}
$$

Expression (13) discretizing continuity Eq. (1) is in all respects a standard finite volume statement (e.g., Toro 1989). Water discharge $Q_{i+1 / 2}^{*}$ exiting cell $i$ is equivalent to discharge $Q_{i+1-1 / 2}^{*}$ entering cell $i+1$, hence strict conservation is enforced. Expression (14) discretizing our modified momentum balance Eq. (12), on the other hand, represents a departure from classical finite volume formulations. The momentum flux $\Sigma_{i+1 / 2}^{*}$ is no longer equivalent when seen from the left or from the right of a given interface $i+1 / 2$. In general, $\Sigma_{i+1 / 2}^{*} \neq \Sigma_{i+1 / 2}^{*}$, right and the difference stems entirely from term $-\left.g I_{1}\right|_{\bar{z}}$ accounting for the effect of variable channel geometry (inclination and nonprismaticity combined). This reflects the nonconservative nature of topographic effects: Momentum is fed into or drained from the flow due to the pressure thrust exerted on its rigid boundaries. Consistent with modified Eq. (12), we account for this nonconservative behavior by making the flux terms asymmetric rather than by adding a topographic source term. It is proposed to refer to this asymmetric evaluation of the momentum flux by the term "flux lateralization."

\section{Characteristic-Based Flux Predictor}

To complete statements (13) and (14), it is necessary to evaluate fluxes $Q_{i+1 / 2}^{*}, \Sigma_{i+1 / 2}^{*}$, left and $\Sigma_{i+1 / 2}^{*}$,right at each interface $i+1 / 2$. This is performed by extending the characteristic-based flux predictor of Braschi and Gallati (1992). Equations in characteristic form must first be obtained by combining Eqs. (1) and (12) and isolating total derivatives $d / d t=\partial / \partial t+d x / d t \cdot \partial / \partial x$. This yields the following characteristic equations:

$$
\frac{d Q}{d t}=c(\mathrm{~F} \mp 1) \frac{d A}{d t}+\frac{c}{\mathrm{~F} \pm 1} \frac{\left.d A\right|_{\bar{z}}}{d t}
$$

valid along characteristic paths $\Lambda_{-}$and $\Lambda_{+}$defined by $\Lambda_{ \pm} \equiv d x / d t=V \pm c$, where $\mathrm{F}=V / c=$ Froude number; $c$ $=(g A / B)^{1 / 2}=$ celerity of hydrodynamic perturbations; and $B=$ free surface width. As illustrated in Fig. 3, the procedure of 


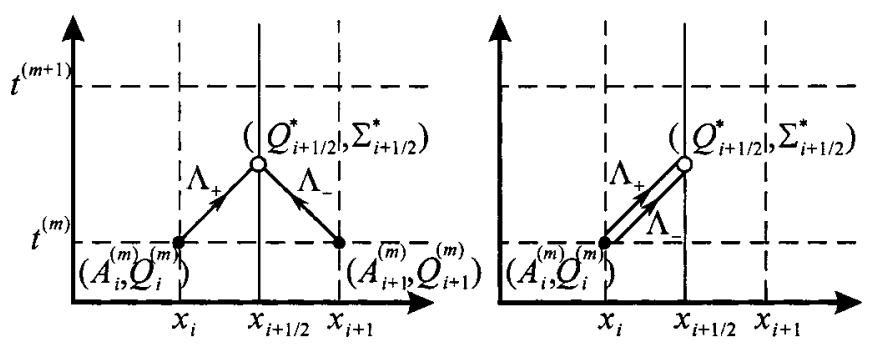

Fig. 3. Approximate characteristic paths adopted for formulation of flux-predictor scheme (Braschi and Gallati 1992). Left: subcritical case $\overline{\mathrm{F}} \leqslant 1$; right: supercritical case $\overline{\mathrm{F}}>1$.

Braschi and Gallati evaluates fluxes $Q_{i+1 / 2}^{*}$ by approximating characteristic Eqs. (16) in the following way. Average values of $F$ and $c$ are first introduced as

$$
\begin{gathered}
\overline{\mathrm{F}}=\overline{\mathrm{F}}_{i+1 / 2}=\frac{V_{i}^{(m)}+V_{i+1}^{(m)}}{c_{i}^{(m)}+c_{i+1}^{(m)}} \\
\bar{c}=\bar{c}_{i+1 / 2}=1 / 2\left(c_{i}^{(m)}+c_{i+1}^{(m)}\right)
\end{gathered}
$$

Let $\bar{F} \geqslant 0$ without loss of generality (the reverse flow case $\bar{F} \leqslant 0$ is treated in a symmetric fashion). If $\bar{F} \leqslant 1$, the flow is subcritical and characteristics $\Lambda_{+}$and $\Lambda_{-}$originate from opposite sides of the interface. One may therefore approximate

$$
\begin{aligned}
Q_{i+1 / 2}^{*}-Q_{i}^{(m)}= & \bar{c}(\overline{\mathrm{F}}-1)\left(A_{i+1 / 2}^{*}-A_{i}^{(m)}\right) \\
& +\frac{\bar{c}}{\overline{\overline{\mathrm{F}}}+1}\left(\left(\left.A\right|_{\bar{z}}\right)_{i+1 / 2}^{*}-\left.A_{i}\right|_{\bar{z}}\right) \\
Q_{i+1 / 2}^{*}-Q_{i+1}^{(m)}= & \bar{c}(\overline{\mathrm{F}}+1)\left(A_{i+1 / 2}^{*}-A_{i+1}^{(m)}\right) \\
& +\frac{\bar{c}}{\overline{\mathrm{F}}-1}\left(\left(\left.A\right|_{\bar{z}}\right)_{i+1 / 2}^{*}-\left.A_{i+1}\right|_{\bar{z}}\right)
\end{aligned}
$$

where $\bar{z}=1 / 2\left(z_{i}^{(m)}+z_{i+1}^{(m)}\right)$. Eliminating $A_{i+1 / 2}^{*}$ between Eqs. (18) and (19) yields statement

$$
\begin{aligned}
Q_{i+1 / 2}^{*}= & \frac{1+\overline{\mathrm{F}}}{2} Q_{i}^{(m)}+\frac{1-\overline{\mathrm{F}}}{2} Q_{i+1}^{(m)}+\frac{\bar{c}}{2}\left(1-\overline{\mathrm{F}}^{2}\right)\left(A_{i}^{(m)}-A_{i+1}^{(m)}\right) \\
& -\frac{\bar{c}}{2}\left(\left.A_{i}\right|_{\bar{z}}-\left.A_{i+1}\right|_{\bar{z}}\right)
\end{aligned}
$$

On the other hand, when $\overline{\mathrm{F}}>1$, the flow is supercritical and both characteristics originate from upstream. While this implies control by upstream flow conditions alone, it is nonetheless necessary to account for topography variations between the upstream and downstream sections. These two considerations lead to statement

$$
Q_{i+1 / 2}^{*}=Q_{i}^{(m)}-\frac{\bar{c}}{2}\left(\left.A_{i}\right|_{\bar{z}}-\left.A_{i+1}\right|_{\bar{z}}\right)
$$

which coincides with Eq. (20) when $\bar{F}=1$. In the absence of the second term on the right-hand side of Eq. (21), spurious discontinuities would arise at critical sections.

In the absence of topography variations, statements (20) and (21) are identical to the original expressions proposed by Braschi and Gallati (1992). Aside from averages (17), the algorithm of Braschi and Gallati is also identical to the better-known Roe scheme (Roe 1981; Alcrudo et al. 1992; Glaister 1993). The present work refers to the former rather than the latter because the derivation of Braschi and Gallati (1992) retains a closer connection with the method of characteristics. This makes extensions to variable topography somewhat more intuitive. Regardless of the averages chosen, statement (20) represents an "upwinding" of the flux estimate: Depending on the Froude number, the predicted discharge is weighted more heavily towards the upstream value $Q_{i}^{(m)}$ than towards the downstream value $Q_{i+1}^{(m)}$. This is a general feature of the so-called "upwind schemes," guaranteeing that the propagation of information is consistent with the characteristic directions. Upwinding prevents the development of nonphysical oscillations in the vicinity of shocks, but also introduces considerable artificial dissipation. To reduce artificial dissipation while retaining nonoscillatory properties, it is necessary to resort to higher-order schemes equipped with flux or slope limiters (e.g., Glaister 1993, Nujic 1995, Sanders 2001) or with a more selective dissipation operator (e.g., Meselhe et al. 1997). In the present work, such extensions are not contemplated and only a first-order accurate formulation is examined.

Corresponding expressions for fluxes $\Sigma_{i+1 / 2}^{*}, \sum_{i+1 / 2}^{*, \text { right }}$ derive from a similar procedure. Using chain rule manipulations, characteristic Eqs. (16) are rewritten in the equivalent form

$$
\frac{d}{d t}\left(\frac{Q^{2}}{A}+g I_{1}-\left.g I_{1}\right|_{\bar{z}}\right)=c(\mathrm{~F} \mp 1) \frac{d Q}{d t}
$$

Upon discretizing these characteristic equations in the same way as before, one obtains

$$
\begin{aligned}
& \text { If } \bar{F} \leqslant 1 \text {, } \\
& \sum_{i+1 / 2}^{*, \mathrm{left}}=\frac{1+\overline{\mathrm{F}}}{2} \frac{Q_{i}^{(m) 2}}{A_{i}^{(m)}}+\frac{1-\overline{\mathrm{F}}}{2}\left(\frac{Q_{i+1}^{(m) 2}}{A_{i+1}^{(m)}}+g\left(I_{1}\right)_{i+1}^{(m)}-\left(\left.g I_{1}\right|_{\left.z_{i}^{(m)}\right)_{i+1}}\right)\right. \\
& +\frac{\bar{c}}{2}\left(1-\overline{\mathrm{F}}^{2}\right)\left(Q_{i}^{(m)}-Q_{i+1}^{(m)}\right) \\
& \sum_{i+1 / 2}^{*, \text { right }}=\frac{1+\overline{\mathrm{F}}}{2}\left(\frac{Q_{i}^{(m) 2}}{A_{i}^{(m)}}+\left(g I_{1}\right)_{i}^{(m)}-\left(\left.g I_{1}\right|_{z_{i+1}^{(m)}}\right)_{i}\right)+\frac{1-\overline{\mathrm{F}}}{2} \frac{Q_{i+1}^{(m) 2}}{A_{i+1}^{(m)}} \\
& +\frac{\bar{c}}{2}\left(1-\overline{\mathrm{F}}^{2}\right)\left(Q_{i}^{(m)}-Q_{i+1}^{(m)}\right)
\end{aligned}
$$

If $\overline{\mathrm{F}}>1$,

$$
\begin{gathered}
\sum_{i+1 / 2}^{*, \text { left }}=\frac{Q_{i}^{(m) 2}}{A_{i}^{(m)}} \\
\sum_{i+1 / 2}^{*, \text { right }}=\frac{Q_{i}^{(m) 2}}{A_{i}^{(m)}}+\left(g I_{1}\right)_{i}^{(m)}-\left(\left.g I_{1}\right|_{z_{i+1}^{(m)}}\right)_{i}
\end{gathered}
$$

where expression $\left(g I_{1} \mid z_{i+1}^{(m)}\right)_{i}$ represents a pressure thrust to be evaluated according to the cross-section data of position $x_{i}$, but with the free surface elevation $z_{i+1}^{(m)}$ sampled at position $x_{i+1}$. Statements (23) share a similar structure with Eqs. (20) and (21), and are upwinded in the same way. Motivation for the present treatment of topography terms is apparent from the examination of Eqs. (20), (21), and (23): All terms involving the channel geometry (the various $A$ 's and $I_{1}$ 's) are sampled only where the geometry is defined, i.e., at sections $x_{i}, x_{i+1}$. Geometry variations are measured by sampling the cross-sectional profile at one location (e.g., $x_{i}$ ), while taking the reference free surface level from 
another location (e.g., $x_{i+1}$ ). This conjugate sampling is the key device involved in constructing the lateralized flux predictors.

\section{Treatment of Critical Sections and Wetting Fronts}

Statements (13) and (14) associated with flux predictors (20), (21), and (23) constitute the core of the numerical scheme proposed in the present work. As such, however, the scheme is not yet fully suitable for general applications. There remain two singularities which require a special treatment: critical sections and wetting fronts. When the flow evolves from a subcritical $(F<1)$ to a supercritical regime ( $F>1)$, a spurious shock is generated by the basic scheme above and other similar algorithms. The second singularity occurs at moving boundaries separating the dry and wet reaches of a channel. In the absence of topography variations, the two singularities can be handled by appending the following exceptions to statement (17):

$$
\begin{aligned}
& \text { If } \mathrm{F}_{i}^{(m)}<1 \text { and } \mathrm{F}_{i+1}^{(m)}>1 \text {, then } \overline{\mathrm{F}}=\mathrm{F}_{i}^{(m)}, \quad \bar{c}=\bar{c}_{i}^{(m)} \\
& \text { If } h_{i}^{(m)}>h_{\min } \text { and } h_{i+1}^{(m)}<h_{\min }, \text { then } \overline{\mathrm{F}}=\mathrm{F}_{i}^{(m)}, \quad \bar{c}=\bar{c}_{i}^{(m)}
\end{aligned}
$$

Exception (24) insures that some downstream influence is felt at the critical section. In dry bed exception (25), $h_{\min }$ is a small cutoff depth, typically of the order of $h_{0} / 100$ where $h_{0}$ is a characteristic depth of the flow. The two exceptions (24) and (25) can be seen as local applications of the Harten-Lax-Van Leer (HLL) scheme of Harten et al. (1983).

The situation becomes more complicated in the presence of topography variations. For a sloping channel, it may happen that at some locations $z_{0 i}>z_{i+1}$, i.e., the bed elevation at one section is higher than the water free surface elevation at an adjacent section. In such cases, negative depths are encountered in Eq. (23) when performing the conjugate sampling of cross-sectional profiles and free surface elevations. To deal with these circumstances, care must be taken in choosing the reference free surface elevations appropriate for the conjugate sampling. This and the other exceptions above are handled in the operational version of the proposed algorithm given in the Appendix.

As the scheme is explicit, the usual Courant-Friedrichs-Lewy (CFL) constraint on the time step must be enforced, i.e.

$$
\mathrm{Cr}=\Delta t \max _{i}\left(\frac{\left|V_{i}\right|+c_{i}}{(\Delta x)_{i}}\right)<1
$$

where $\mathrm{Cr}=$ Courant number. All computations reported below were performed with $\mathrm{Cr}=0.9$.

\section{External and Internal Boundary Conditions}

Specific treatments are required to deal with the external and internal boundaries of river channel networks. For boundaries where the flow is subcritical, external data such as prescribed discharges or water levels are combined with a one-sided version of the flux predictor algorithm. Supercritical boundaries involve either two external conditions (inflow) or a fully upwind version of the flux predictor (outflow). Such treatment of external boundaries is described in detail in Capart et al. (1999). Junctions between multiple channels, on the other hand, form internal boundaries. Each junction is treated as a finite volume of its own, to which a continuity equation and a $2 \mathrm{D}$ version of balance law (11) are applied. At the interfaces which connect the various channels to the junction control volume, the lateralized flux predictor algorithm is applied without modifications.
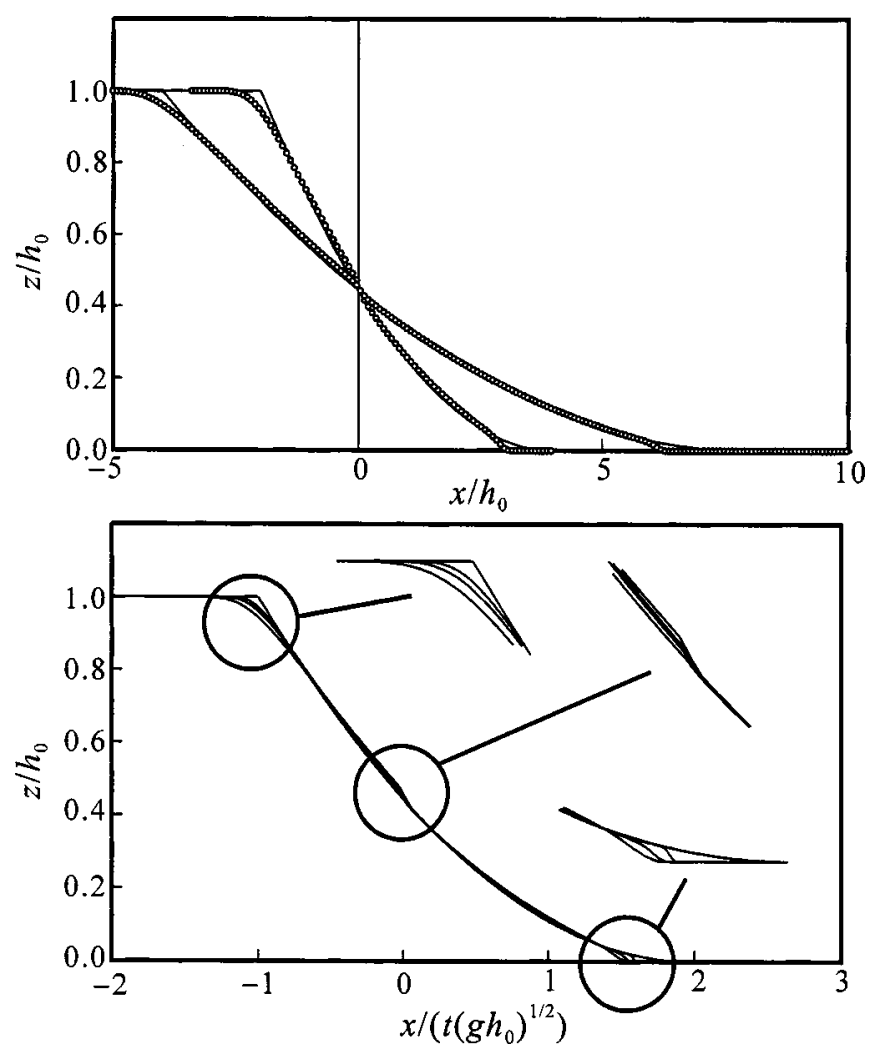

Fig. 4. Dam-break wave over a dry bed. Top: comparison of computational results (circles) with Ritter analytical solution (continuous lines). Bottom: convergence of computational results toward exact solution.

\section{Model Verification}

\section{Dam-Break Wave Over Dry Bed}

Before testing the proposed scheme in the presence of geometry variations, the algorithm is first applied to a horizontal prismatic channel. We choose as a test case the well-known dam-break problem. Motionless water of constant depth $h_{0}$ is held upstream of a dam, then instantaneously released over a dry, frictionless bed. An analytical solution for the resulting flow is the wellknown Ritter (1892) centered simple wave, which exhibits a critical section at the initial dam position $(x=0)$. Featuring transcritical and wetting front singularities, this case is often used as a benchmark test for transient shallow water solvers. Fig. 4 (top) compares computational results with the exact solution at nondimensional times $t /\left(h_{0} / g\right)^{1 / 2}=2$ and 4 . The first-order scheme introduces artificial diffusion and smoothes out the sharp cusp presented by the analytical solution at the upstream edge of the wave. The computed free surface further features a slight twist at the critical section, and deforms into a borelike bulge of small depth at the wavefront. Overall, however, satisfactory agreement is recorded despite the coarse resolution adopted for illustration purposes. Fig. 4 (bottom) shows how computational results converge towards the exact solution as the discretization is refined (spatial and time steps twice decreased by a factor of 2). Details of the more sensitive regions are enlarged. Globally, the computations appear on a par with results from similar finite volume algorithms (e.g., the Roe method) and other numerical schemes documented in the literature (e.g., Glaister 1993; Ambrosi 1995; Khan 2000). When propagating shocks are present, similar com- 


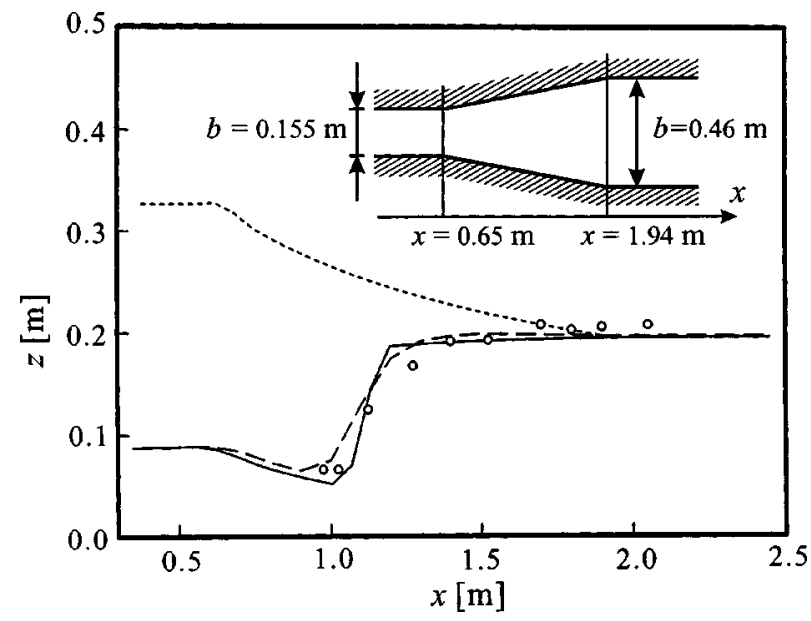

Fig. 5. Centerline profiles for hydraulic jump in channel expansion. Inset: definition sketch; circles: experimental measurements (Khalifa 1980); long dashes: 2D shallow water computations of Younus and Chaudhry (1994); continuous line: present 1D results; short dashes: scheme of Braschi and Gallati (1992) without topography corrections.

putations compare favorably with analytical profiles, experimental data, and results from other numerical schemes, as documented in Braschi and Gallati (1992) and Pagliara et al. (1999).

\section{Hydraulic Jump in Channel Expansion}

The formation of a hydraulic jump in a divergent channel is examined as a second test case. The channel has a horizontal bottom and rectangular cross sections, with a nonprismatic geometry shown on Fig. 5 (inset). The inlet flow is supercritical and characterized by discharge $Q=0.0263 \mathrm{~m}^{3} / \mathrm{s}$ and depth $h=0.088 \mathrm{~m}$. A hydraulic jump is forced into the channel by imposing a downstream water depth $h=0.195 \mathrm{~m}$. Experimental measurements for this flow were obtained by Khalifa (1980) and used by Younus and Chaudhry (1994) to test a 2D shallow water scheme. This application checks the ability of the scheme to deal with nonprismatic effects in the presence of a shock. The surface profiles computed using the present 1D scheme are compared on Fig. 5 with centerline data from the experiments and 2D computations. By taking nonprismaticity into account, the present algorithm is able to accurately predict the location and magnitude of the jump, at a fraction of the computational cost incurred by $2 \mathrm{D}$ schemes. For comparison, Fig. 5 also includes a computed profile for which the topography correction was deactivated. The result is way off, both quantitatively and qualitatively (the hydraulic jump migrates upstream and drowns the supercritical jet at the inlet). This emphasizes the importance of the hydrostatic pressure contributions acting on nonprismatic channel boundaries.

\section{Tanshui Case Study}

\section{Tanshui River and Herb Typhoon Flood}

The approach can now be applied to the practical case which motivated its development. Flowing into the Taiwan Strait, the Tanshui River drains a highly urbanized basin encompassing most of the Taipei metropolitan area (Fig. 6). The river receives inflows from two torrential streams, the Tahan and Shindian Creeks, and from the mild-sloped Keelung River. Average discharges for these three tributaries are respectively $62.1,72.7,26.1 \mathrm{~m}^{3} / \mathrm{s}$ (Liu et al. 2001). A diversion channel created to increase conveyance and mitigate flood hazards runs alongside a portion of the Tanshui, giving the network a looped topology. Tidal influence reaches as far as the foot of the Tahan and Shindian Creeks, where the water surface presents a sharp change in slope. Mountainous streams and tidal estuary are thus combined in a very compact watershed.

A number of 1D dynamic flood routing methods have been applied to the Tanshui River system. These include the implicit finite difference Preissmann four-point scheme (Yen and Hsu 1984), finite elements (Young 1991) and the multimode method of characteristics (Lai 1988). Application of these schemes has

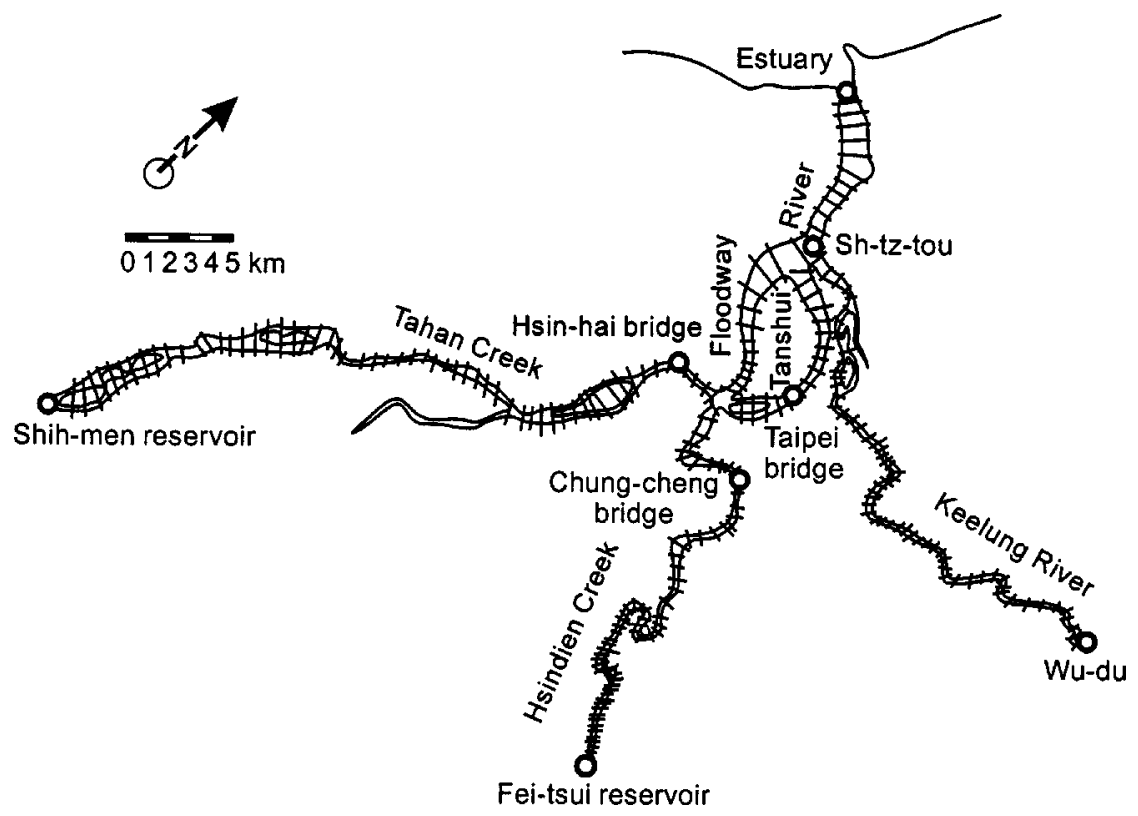

Fig. 6. Diagram of the Tanshui River network showing the various channels, surveying transects, and gaging stations 
however been limited to a truncated version of the river network, leaving out the upper reaches. The reason is that the above treatments could deal neither with transitions between supercritical and subcritical regimes of flow, nor with the dry bed problem. Both difficulties are encountered upstream of the Tanshui network: The former when torrential flows from the steep-sloped creeks meet the tidal backwater, the latter in the absence of reservoir outflow feeding the upper Tahan and Shindian. Channel geometry data are available as cross-section profiles surveyed along the transects shown in Fig. 6. Typical profiles are plotted in Fig. 1 for the Tahan Creek and Tanshui River, illustrating the highly irregular bathymetry. Combining transcritical flow and abrupt topography, the Tanshui network exhibits the full range of conditions which the present method seeks to address.

The proposed scheme is applied to an exceptionally severe flood recorded in 1996. As Typhoon Herb struck Taiwan on July 31 , 1996, a record rainfall of 2,000 $\mathrm{mm}$ poured over the Tanshui basin over a two-day period. As a consequence, both the Shihmen and Feitsui reservoirs had to release large volumes of water to the Tahan and Shindian Creeks. Conveyed downstream, the storm flood inundated some of Taipei's most densely settled areas, affecting an estimated 3,550 homes. Two test runs are presented, corresponding to two different sets of boundary conditions. The first run simulates flow through the full river network and takes its boundary conditions from as far upstream as possible, including discharge hydrographs measured at the Shihmen and Feitsui reservoirs. This tests the ability of the model to deal with a combination of subcritical, supercritical, and dry reaches. By imposing conditions far upstream, however, some nonrecorded discharges from lesser tributaries are missed. In order to make a more precise comparison with the observed flood levels, the second run truncates the network and samples boundary conditions further downstream. The discharge hydrographs at the reservoir spillways are replaced by water stage hydrographs given at Hsin-hai bridge and Chung-cheng bridge along the Tahan and Shindian Creeks. For both runs, the remaining boundary conditions are imposed as water stage hydrographs recorded downstream at the river mouth and upstream of the Keelung River at Wu-du station. Additional water stage hydrographs are measured at the Taipei bridge and Sh-tz-tou. The locations of the various stations are indicated on Fig. 6.

\section{Results and Discussion}

Data and computational results for the 1996 flood are presented in Figs. 7-9. The hydrographs and limnigraphs used as boundary conditions are plotted in Fig. 7. Two distinct phases of the simulation can be distinguished. In the first phase, the typhoon flood has not yet been felt and tidal influence constitutes the dominant effect. No inflows are yet received from the Shihmen and Feitsui reservoirs, hence the upstream Tahan and Shindian Creeks run partially dry. For this phase, it matters little which set of boundary conditions is used. Typical water profiles are shown in Fig. 8. The Tanshui estuary and Tahan Creek (top panel of Fig. 8) exhibit nearly horizontal water surfaces. In the absence of significant inflow, what is tested is mainly the ability of the scheme to equilibrate water at rest and deal with submergence and emergence of channel reaches as the tide flows in and out. The Keelung river (bottom panel of Fig. 8), on the other hand, does receive some inflow and presents a mildly sloped free surface. Good results are obtained. Despite the abrupt topography, gradually varied water profiles are computed. This is consistent with the expected behavior for this low Froude number situation. In the absence of a

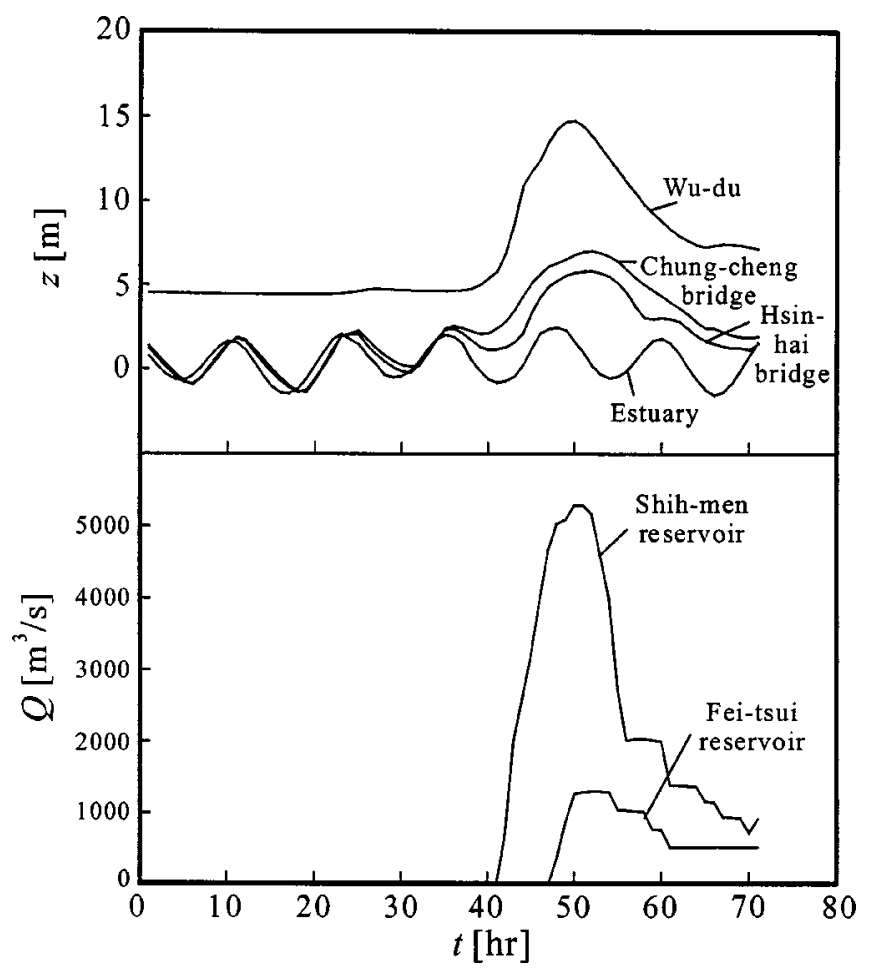

Fig. 7. Boundary conditions for Herb typhoon flood of 1996. Top: water stage hydrographs; bottom: discharge hydrographs at reservoir outlets.

careful momentum balance accounting for geometry variations, highly irregular free surface profiles would be obtained in the same configuration (Nujic 1995). In Fig. 8 (top), the transition from dry bed to submerged conditions at the limit of tidal influence is also seen to be well captured.

As the typhoon hits, large discharges are released from the two reservoirs into the Tahan and Shindian Creeks, and the discharge into the Keelung River increases as well. Profiles of the maximum water levels attained during the flood are shown in Fig. 8. Water profiles typical of supercritical flows are computed in the upper reaches of the Shindian and Tahan Creeks. Due to local accidents in topography (sills and constrictions), transitions between superand sub-critical flow are observed at various locations. Further downstream, flatter, more regular free surface profiles characterize the flow which becomes controlled by the tidal backwater effect. Well-behaved results are again obtained despite the abrupt topography. Computed for full network conditions, the numerical profiles of Fig. 8 are in reasonable agreement with the recorded water levels. Significantly, the discrepancies in water level are verified to be small with respect to the typical amplitude of the bathymetry variations. For the fluvial reaches, this demonstrates the ability of the scheme to obtain good results despite the abrupt character of the valley. For the torrential reaches of the steeper upstream channels, however, the description is not expected to be so accurate. Where steep free surface inclinations are combined with abrupt width variations, the error associated with momentum balance approximation (10) becomes more significant.

Fig. 9 compares the predicted and measured stage hydrographs recorded at the Taipei Bridge (location of the station is shown in Fig. 6). Results for the tidal-dominated phase are seen to be in good agreement regardless of the boundary conditions adopted. During the passage of the typhoon flood wave, results for the full network (discharge boundary conditions imposed far upstream) 

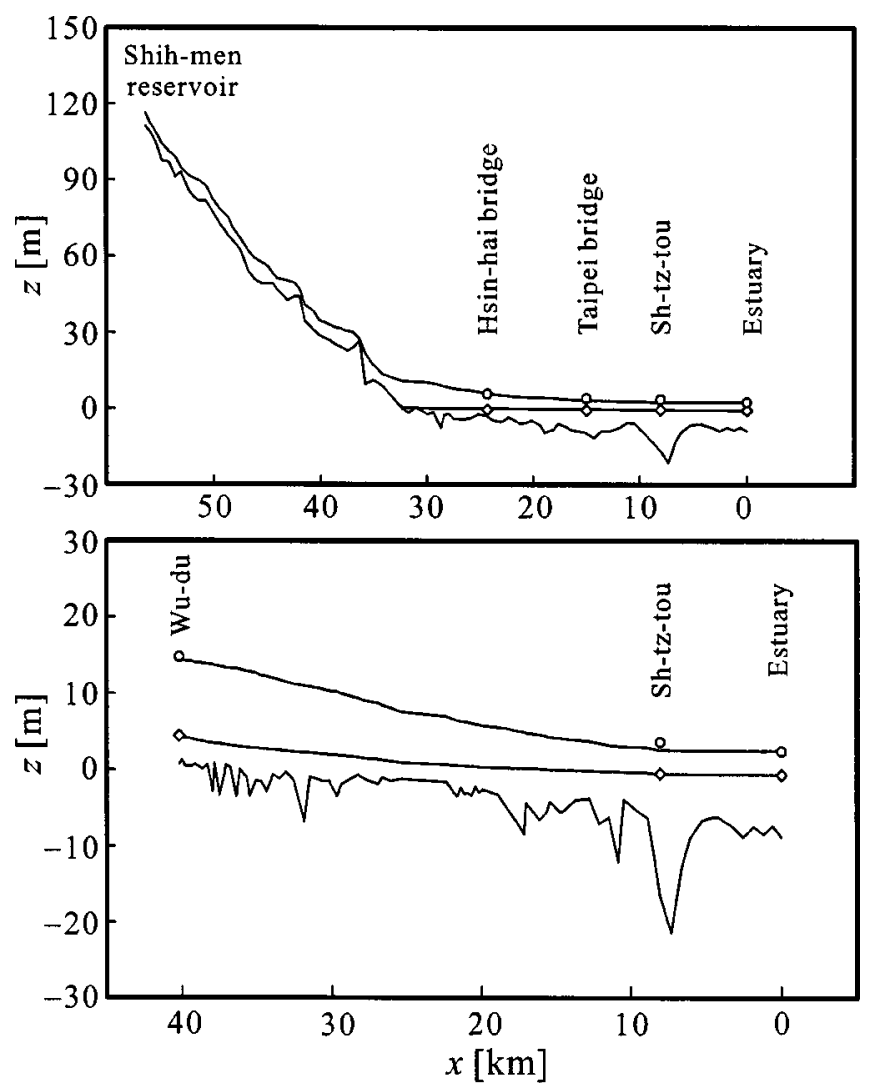

Fig. 8. Longitudinal profiles along Tahan Creek and Tanshui River (top) and along Keelung River (bottom). Continuous lines: from bottom to top, surveyed river bottom profile, computed water profile at time $t=4 \mathrm{~h}$, and computed maximum water profile attained during Herb typhoon flood. Symbols: corresponding gauge measurements.

are not as good. Peak elevations are underpredicted, and the falling limb of the flood wave is overpredicted. These discrepancies are attributed in part to additional discharges from lesser tributaries which are missed by the upstream boundary conditions. It is likely that deficiencies in the frictional model are also to blame. As these impact the stage-discharge relation, water surface profiles are affected more strongly when prescribing discharges than when imposing water levels at the boundaries. In the simulations, a constant Manning coefficient $\left(n=0.02-0.025 \mathrm{~s} / \mathrm{m}^{1 / 3}\right.$ depending

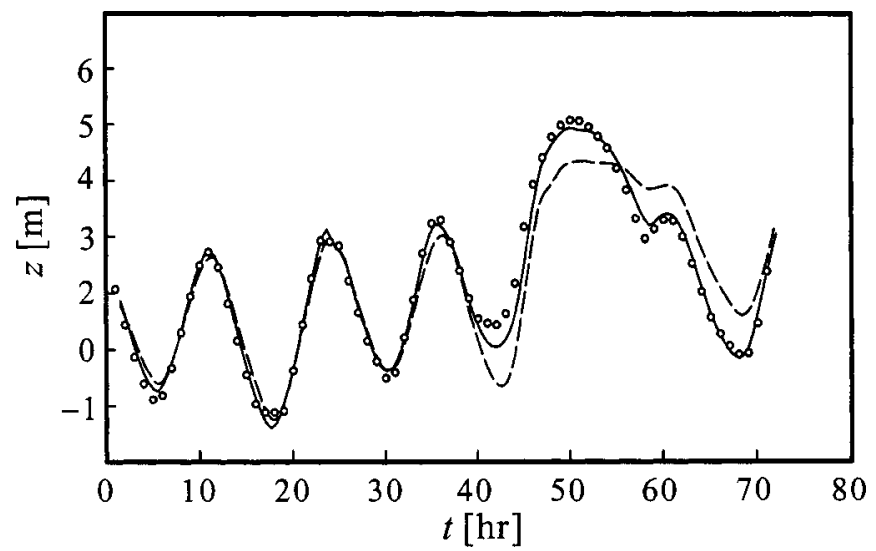

Fig. 9. Water stage hydrographs at Taipei bridge. Circles: gauge measurements; dashed lines: full network computations; continuous lines: truncated network computations.

392 / JOURNAL OF HYDRAULIC ENGINEERING @ ASCE / MAY 2003 on the reach) is used along the channel perimeter. Better agreement could possibly be obtained by considering the effect of compound channel flow, using for example the practical approach proposed by Bousmar and Zech (1999). By contrast, results are much better for the truncated network (water stage boundary conditions imposed further downstream). The above effects have a lesser influence, and excellent agreement is recorded between computations and measurements.

\section{Summary and Conclusions}

As illustrated by the Tanshui case study, practical river computations must often deal with multiple flow regimes in channels of highly irregular bathymetry. Standard finite volume schemes are well equipped to deal with shocks and various types of flow transitions. They are less apt at balancing the hydrostatic pressure contributions due to geometry variations. The present work seeks to address this problem while retaining the unique advantages of finite volume schemes. To do so, a modified version of the SaintVenant momentum equation is introduced. This equation is then used to formulate topographic corrections to the upwind algorithm of Braschi and Gallati (1992). The resulting scheme performs well in idealized verification tests. Good results are also obtained for a challenging river network subject to severe flood conditions. To conclude, the Tanshui River results indicate that the proposed finite volume scheme can be successfully applied to the routing of floods in natural valleys. Starting with the Roe scheme (for which only a change of averages is required), it is likely that the present approach can be extended to other numerical methods. Further developments are however required to improve the treatment of steep-sloped reaches, go beyond first-order accuracy, and endow the topographic corrections with a more rigorous theoretical basis.

\section{Acknowledgments}

Valuable insights regarding the issue of topography in finite volume schemes were gained from Xavier Sillen and Sandra Soares Frazao, Univ. catholique de Louvain, Belgium. Support of this work by the National Science Council, Taiwan, is also greatly appreciated.

\section{Appendix: Lateralized Flux Predictor Algorithm}

Handling the various exceptions, the operational algorithm proceeds in four steps:

- Step 1: Computation of the averages $\overline{\mathrm{F}}, \bar{c}$ using Eq. (17) and exceptions (24) and (25).

- Step 2: Flux predictor algorithm of Braschi and Gallati (1992) If $\bar{F} \leqslant 1$ :

$$
\begin{aligned}
& Q_{i+1 / 2}^{*}= \frac{1+\overline{\mathrm{F}}}{2} Q_{i}^{(m)}+\frac{1-\overline{\mathrm{F}}}{2} Q_{i+1}^{(m)}+\frac{\bar{c}}{2}\left(1-\overline{\mathrm{F}}^{2}\right)\left(A_{i}^{(m)}-A_{i+1}^{(m)}\right) \\
& \Sigma_{i+1 / 2}^{*}= \frac{1+\overline{\mathrm{F}}}{2}\left(\frac{Q_{i}^{(m) 2}}{A_{i}^{(m)}}+\left(g I_{1}\right)_{i}^{(m)}\right) \\
&+\frac{1-\overline{\mathrm{F}}}{2}\left(\frac{Q_{i+1}^{(m) 2}}{A_{i+1}^{(m)}}+\left(g I_{1}\right)_{i+1}^{(m)}\right) \\
&+\frac{\bar{c}}{2}\left(1-\overline{\mathrm{F}}^{2}\right)\left(Q_{i}^{(m)}-Q_{i+1}^{(m)}\right) \\
& \text { If } \overline{\mathrm{F}}>1: \quad Q_{i+1 / 2}^{*}=Q_{i}^{(m)}, \quad \sum_{i+1 / 2}^{*}=\frac{Q_{i}^{(m) 2}}{A_{i}^{(m)}}+\left(g I_{1}\right)_{i}^{(m)}
\end{aligned}
$$


- Step 3: Computation of average and reference free surface elevations $\bar{z}, \hat{z}_{i}, \hat{z}_{i+1}$

General case: $\bar{z}=\bar{z}_{i+1 / 2}^{*}=1 / 2\left(z_{i}^{(m)}+z_{i+1}^{(m)}\right), \quad \hat{z}_{i}=z_{i}^{(m)}, \quad \hat{z}_{i+1}$ $=z_{i+1}^{(m)}$

Exceptions:

If $h_{i}^{(m)}<h_{\min }$ and $z_{0 i}^{(m)}>z_{i+1}^{(m)}$ then $\bar{z}=\hat{z}_{i}=\hat{z}_{i+1}=z_{i+1}^{(m)}$

If $h_{i+1}^{(m)}<h_{\min }$ and $z_{0 i+1}^{(m)}>z_{i}^{(m)}$ then $\bar{z}=\hat{z}_{i}=\hat{z}_{i+1}=z_{i}^{(m)}$

- Step 4: Topography corrections

$$
\begin{gathered}
Q_{i+1 / 2}^{*}=Q_{i+1 / 2}^{*}-\frac{\bar{c}}{2}\left(\left.A_{i}\right|_{\bar{z}}-\left.A_{i+1}\right|_{\bar{z}}\right) \\
\sum_{i+1 / 2}^{* \text { left }}=\Sigma_{i+1 / 2}^{*}-\frac{1+\overline{\mathrm{F}}}{2}\left(g I_{1}\right)_{i}^{(m)}-\frac{1-\overline{\mathrm{F}}}{2}\left(\left.g I_{1}\right|_{\hat{z}_{i}}\right)_{i+1} \\
\sum_{i+1 / 2}^{*, \text { right }}=\Sigma_{i+1 / 2}^{*}-\frac{1+\overline{\mathrm{F}}}{2}\left(\left.g I_{1}\right|_{\hat{z}_{i+1}}\right)_{i}-\frac{1-\overline{\mathrm{F}}}{2}\left(g I_{1}\right)_{i+1}^{(m)}
\end{gathered}
$$

In these last three statements, zero values are assigned to the $A$ 's and $I_{1}$ 's if they are evaluated for negative depths.

\section{References}

Alcrudo, F., Garcia-Navarro, P., and Saviron, J. M. (1992). "Flux difference splitting for 1D open-channel flow equations." Int. J. Numer. Methods Fluids, 14, 1009-1018.

Ambrosi, D. (1995). "Approximation of shallow water equations by Roe's Riemann Solver.” Int. J. Numer. Methods Fluids, 20, 157-168.

Bermudez, A., and Vasquez, M. E. (1994). "Upwind methods for hyperbolic conservation laws with source terms." Comput. Fluids, 23(8), 1049-1071.

Bousmar, D., and Zech, Y. (1999). "Momentum transfer for practical flow computation in compound channels." J. Hydraul. Eng., 125(7), 696-706.

Bradford, S. F., and Sanders, B. F., (2002). Finite-volume model for shallow-water flooding of arbitrary topography. J. Hydraul. Eng., 128(3), 289-298.

Braschi, G., and Gallati, M. (1992). "A conservative flux prediction algorithm for the explicit computation of transcritical flow in natural streams." Proc., Hydrosoft '92. Hydraulic Engineering Software IV: Fluid Flow Modeling, Computational Mechanics Publications, Southampton, England, 381-395.

Capart, H., Sillen, X., and Zech, Y. (1997). "Numerical and experimental water transients in sewer pipes." J. Hydraul. Res., 35(5), 659-672.

Capart, H., Bogaerts, C., Kevers-Leclercq, J., and Zech, Y. (1999). "Robust numerical treatment of flow transitions at drainage pipe boundaries." Water Sci. Technol., 39, 113-120.

Chaudhry, M. H. (1993). Open-channel flow, Prentice-Hall, N.J.

Cunge, J. A., Holly, F. M., Jr., and Verwey, A. (1980). Practical aspects of computational river hydraulics, Pitman, London.

Fennema, R. J., and Chaudhry, M. H. (1987). "Simulation of onedimensional dam break flows." J. Hydraul. Res., 25(1), 41-51.

Fraccarollo, L., and Toro, E. F. (1995). "Experimental and numerical assessment of the shallow water model for two-dimensional dambreak type problems." J. Hydraul. Res., 33(6), 843-864.

Glaister, P. (1988). "Approximate Riemann solutions of the shallow water equations." J. Hydraul. Res., 26(3), 293-306.

Glaister, P. (1993). "Flux difference splitting for open-channel flows." Int. J. Numer. Methods Fluids, 16, 629-654.

Harten, A., Lax, P. D., and Van Leer, B. (1983). "On upstream differenc- ing and Godunov-type schemes for hyperbolic conservation laws." SIAM Rev., 25, 35-61.

Jha, A., Akiyama, J., and Ura, M. (1996). "A fully conservative Beam and Warming scheme for transient open channel flows." J. Hydraul. Res., 34(5), 605-621.

Jin, M., and Fread, D. L. (1997). "Dynamic flood routing with explicit and implicit numerical solution schemes." J. Hydraul. Eng., 123(3), 166-173.

Khalifa, A. M. (1980). "Theoretical and experimental study of the radial hydraulic jump." $\mathrm{PhD}$ thesis, Univ. of Windsor, Windsor, Ont., Canada.

Khan, A. A. (2000). "Modeling flow over an initially dry bed." J. Hydraul. Res., 38(5), 383-388.

Lai, C. (1988). "Comprehensive method of characteristics models for flow simulation." J. Hydraul. Eng., 114(9), 1074-1097.

Liu, W.-C., Hsu, M.-H., and Kuo, A. Y. (2001). "Investigation of longterm transport in Tanshui River Estuary, Taiwan." J. Waterw., Port, Coastal, Ocean Eng., 127(2), 61-71.

Meselhe, E. A., Sotiropoulos, F., and Holly, F. M. Jr., (1997). "Numerical simulation of transcritical flow in open channels." J. Hydraul. Eng., 123(9), 774-783.

Nujic, M. (1995). "Efficient implementation of non-oscillatory schemes for the computation of free-surface flows." J. Hydraul. Res., 33(1), 101-111.

Pagliara, S., Venutelli, M., Capart, H., Sillen, X., and Zech, Y. (1999). Numerical and experimental water transients in sewer pipesDiscussion. J. Hydraul. Res., 37(5), 712-714.

Ritter, A. (1892). "Die fortpflanzung der wasserwellen.” Zeitschr. Ver. Deut. Ing., 36, 947-954.

Roe, P. L. (1981). “Approximate Riemann solvers, parameter vectors, and difference schemes." J. Comput. Phys., 43, 357-372.

Sanders, B. F. (2001). "High-resolution and non-oscillatory solution of the St. Venant equations in non-rectangular and non-prismatic channels." J. Hydraul. Res., 39(3), 321-330.

Sanders, B. F., and Katopodes, N. D. (1999). "Active flood hazard mitigation. I: Bidirectional wave control.” J. Hydraul. Eng., 125(10), 1057-1070.

Soares Frazao, S., Morris, M., and Zech, Y. (2000). Concerted Action on Dambreak Modelling: Objectives, Project Report, Test Cases, Meeting Proceedings. Univ. catholique de Louvain, Civil Engineering Dept., Hydraulics Division, Louvain-la-Neuve, Belgium, CD-ROM.

Toro, E. F. (1989). Riemann solvers and numerical methods for fluid dynamics, Springer, Berlin.

Toro, E. F. (1992). "Riemann problems and the WAF method for solving the two-dimensional shallow water equations." Philos. Trans. R. Soc. London, Ser. A, A338, 43-68.

Tseng, M.-H., and Chu, C. R. (2000). "Two-dimensional shallow water flows simulation using TVD-MacCormack Scheme." J. Hydraul. Res., 38(2), 123-131.

Tseng, M.-H., Hsu, C.-A., and Chu, C. R. (2001). "Channel routing in open-channel flow with surges.” J. Hydraul. Eng., 127(2), 115-122.

Yang, J. Y., Hsu, C. A., and Chang, C. H. (1993). "Computation of free surface flows. Part I: One-dimensional dam-break flow." J. Hydraul. Res., 31(1), 19-34.

Yen, C. L., and Hsu, M. H. (1984). "Flood routing models for Tanshui river system under present condition." Proc., CCNAA-AIT Joint Seminar on Research for Multiple Hazard Mitigation, Tainan, Taiwan, $368-380$.

Young, D. L. (1991). "Finite element modeling of shallow water wave equations." J. Chin. Inst. Eng., 14(2), 143-155.

Younus, M., and Chaudhry, M. H. (1994). "A depth-averaged k- $\epsilon$ turbulence model for the computation of free-surface flow." J. Hydraul. Res., 32(3), 415-444. 